\title{
Fluctuations in quantum dot charging energy
}

\author{
M. Stopa \\ RIKEN (The Institute of Physical and Chemical Research) 2-1, Hirosawa, Wako-Shi Saitama 351-01, Japan \\ e-mail stopa@sisyphus.riken.go.jp
}

(April 22, 2017)

\begin{abstract}
We demonstrate that enhanced fluctuations in the charging energy of chaotic semiconductor quantum dots result from strongly scarred wave functions. We demonstrate that strong scars linger at the Fermi surface due to their enhanced Coulomb interaction between spin up and spin down states. We present density functional and spin density functional calculations to elucidate the temperature, dot shape and spin polarization dependencies of the fluctuations.
\end{abstract}

A simple, physically appealing paradigm for the statistical behaviour of the ground state of chaotic, interacting systems does not yet exist. Based on the concept of level repulsion, the behaviour of eigenvalues and vectors of complex systems has been successfully treated for many years via random matrix theory (RMT) [1]. As recent experiments on quantum dots demonstrate however [2, fluctuations in the ground state energy as a function of electron number $N$, are not well described by RMT since the required hypothesis of constant interaction (CI) appears to be violated. On fundamental grounds this failure is to be expected since the ergodicity implicit in RMT is unconstrained by any minimization principle which must be an essential ingredient of any theory of the ground state.

The charging energy $E_{C}$ of a quantum dot, defined below in terms of the ground state energy at differing $N$, provides an important archetype of an interacting system insofar as particle number, disorder [3], the "chaoticity" [1] and size can be varied. Attempts to understand the scale and behaviour of fluctuations in the charging energy in quantum dots have included numerical studies of tight binding Hamiltonians for small particle number [2.5] as well as study of the statistical behaviour of electron-electron interactions in a circular, disordered dot based on the random phase approximation (RPA) for the screened interaction and a sigma model calculation for the eigenfunction correlator [6].

Exact diagonalization studies such as in Refs. [2,5] give valuable insight into trends in the ground state energy, but they are necessarily limited to small particle number and in addition they depend on a reduction of a complicated set of matrix elements to a small set of energy parameters. Reference [6], on the other hand, considers explicitly direct and exchange Coulomb matrix elements, however the distribution of these elements are evaluated using a wave function correlator for a non-interacting disordered system. Consequently there is no minimization principle and no self-consistency in the theory. Furthermore, both the diagonalization approach and the method of Ref. [6] provide only statistical information on the charging energy. Neither method can realistically incorporate a varying gate voltage and therefore the physical process whereby fluctuations occur is unapproachable.
By contrast, a detailed study of the evolving, selfconsistent eigenfunctions, free energy and level structure of a realistic dot via density functional (DF) theory, which we present here, reveals the specific mechanism whereby $E_{C}$ fluctuations deviate from the "RMT+CI"

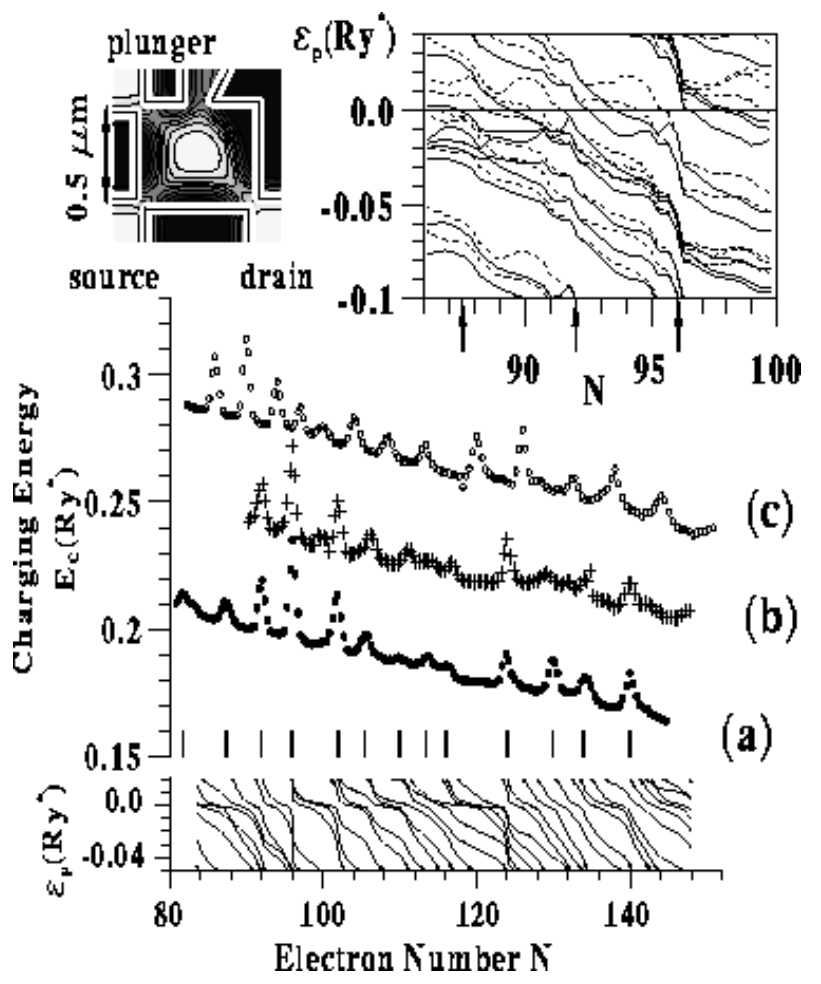

FIG. 1. $E_{C}$ vs. $N$ and spectrum near $E_{F}$ (lower two panels). Curves (a) and (b) differ from (c) by non-plunger gate voltages, hence varied pattern of peaks. (b) and (c) offset by 0.04 and $0.08 R y^{*}$ resp. (a) and (c) are pure Hartree, (b) includes $E_{x c}$ though LDA. $T=0.1 K$ throughout. Spectrum corresponds to (a) and (b). Upper right panel shows levels from SDF calculation, same parameters as (b), arrows indicate $E_{C}$ peaks. Note at $N=92$ and 96 spin polarization of dot collapses. Inset: gate pattern and typical self-consistent effective $2 \mathrm{D}$ potential at $2 \mathrm{DEG}$ level.

predictions. We show that large fluctuations in $E_{C}$ are related to strong scars; remnants of periodic orbits in the classical confining potential. When located at the Fermi surface $E_{F}$ these states, being quasi-one dimen- 
sional, tend to create an inhomogeneous potential, in contrast to other more "chaotic" states which occupy more smoothly the entire dot area. Consequently, when a scar is at the Fermi surface a gap is created to the next state which results in additional gate voltage spacing to the next Coulomb oscillation. Furthermore, we find that the direct Coulomb matrix element between a state and itself (up spin and down spin), denoted $W_{p p} \equiv U_{p}$, is generally greater than between different spatial states $W_{p q}, p \neq q$, and that $U_{p}$ for scars is particularly large. Therefore, as we verify via full spin density functional (SDF) calculations for certain parameter regions, the dot is almost always spin polarized [7]. Further, scars tend to remain, half filled, at $E_{F}$ (as $V_{g}$ is varied) due to their large $U_{p}$, while other, more homogeneous states pass through the Fermi surface [8] (in the process gradually screening the scar state). Finally, when it becomes energetically impossible to draw down a still higher state to $E_{F}$, the filling of the second spin state of the scar does occur, leaving a gap to the next state, as mentioned above. At this point not only the scar, but all states are doubly occupied and spin polarization of the dot collapses.

We calculate the self-consistent electronic structure [3] for a small quantum dot with the wafer profile and gate pattern of the device used by Sivan et al. [2]. The nominal two dimensional electron gas (2DEG) density is $3.1 \times 10^{-11} \mathrm{~cm}^{-2}$. Typical values for the average level spacing (not assuming spin degeneracy) and charging energy are $\Delta \sim 0.012 R y^{*}$ and $E_{C} \sim 0.18 R y^{*}$, respectively (1 $\left.R y^{*} \approx 5.8 \mathrm{meV}\right)$. The gate pattern is rectangular (inset Fig. 1) but due to excess metal in one gate the effective confining potential is classically chaotic, a fact which is substantiated by the statistics of the single particle level spacings (not shown). As in the experiment we vary the "plunger" gate voltage $V_{g}$ (inset Fig. 1), typically in steps of $5 \mathrm{mV}$, the other gates being fixed to isolate the dot from source and drain. For each $V_{g}$ the electronic structure is calculated at three values of dot chemical potential $\mu_{d o t}=0.0, \pm 0.2 R y^{*}$. States are filled (and $N$ thereby determined) according to a Fermi function, which except for very small dots is expected to give a good approximation to the full grand canonical ensemble [9]. Thus, in the calculation $N$ is not necessarily an integer. The values of $\mu_{d o t}$ are chosen to change $N$ by about \pm 1 , relative to the "equilibrium" value $\left(\mu_{\text {dot }}=\mu_{\text {leads }} \equiv 0\right)$. The free energy $F\left(N, V_{g}\right)$ is calculated according to ref. [3] and $E_{C}$ is defined as $\partial^{2} F\left(N, V_{g}\right) / \partial N^{2}$, which we compute discretely.

Traces of $E_{C}(N)$ ( $N$ from $\mu_{d o t}=0$ solution $)$ are shown in Fig. 1. The quasi-periodic peaks are associated with the onset (e.g. $N=87,114$ ) or completion ( $N=92,96,102,124)$ of scar filling, the former being accompanied by downward fluctuation of states below the scar state, the latter with gaps above the scar state. Changing "non-plunger" gates varies the precise pattern of oscillations, but not the basic structure. Varying a "back gate" (not shown) also produces qualitatively similar characteristics.

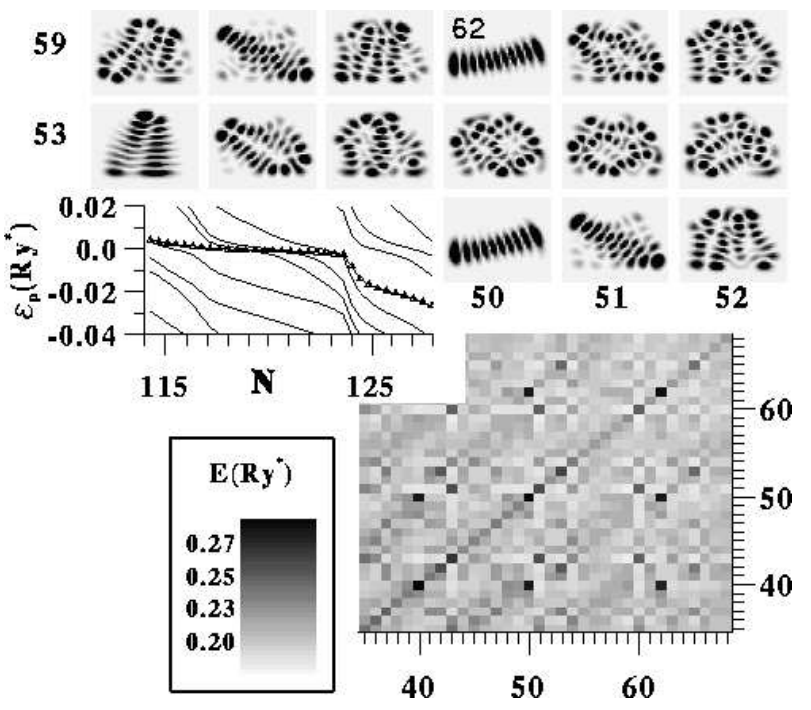

FIG. 2. Selected eigenfunctions (moduli squared) and grey scale of Coulomb matrix elements at $N \approx 124$ (cf. curves (a) and (b) Fig. 1). Inset gives close-up of spectrum from Fig. 1, scar state indicated by triangles remains near $E_{F}$.

Standard DF theory is a spin degenerate mean field theory. Even when exchange-correlation $E_{x c}$ is included in the local density approximation (LDA) it is known that some uncorrected self-interaction remains [10]. The tendency (Fig. 1) for levels to cluster at $E_{F}$ results from the energetic advantage of occupying several states partially rather than one or two states fully. At finite temperature $T$ such partial occupancy can be justified as an approximation to the weighted ensemble of many-body states. Further, in this regime, Wigner crystallization is unimportant. Nonetheless, a more precise description, where the role of spin polarization is made manifest, can be obtained via SDF calculations. As these are numerically more intensive we only calculate the behaviour for various representative parameter regions. Our main physical conclusion is that the electrostatically driven tendency for levels to cluster, partially filled, at $E_{F}$ in ordinary DF theory is replaced by an electrostatically driven spin polarization in SDF (e.g. it is energetically favorable to occupy two different spatial states singly as opposed to occupying either of them doubly).

The spectrum for the SDF case is illustrated in the upper right panel of Fig. 1. The $E_{C}$ fluctuations (not shown) occur at the same $N$ values as in the DF calculations and coincide with the arrows. Note that the fluctuation at $N=87$, which corresponds to the filling of the first spin state of a scar, is qualitatively different from those at $N=92$ and 96 , where scars become doubly occupied. In the latter cases, since $U_{p}$ of the scar is much greater than that of other states, double filling generally only occurs when all other states are either doubly filled 
or empty. Therefore [11], when this state fills, all states of the dot become identically spin degenerate.

Figure 2 shows wave functions and Coulomb matrix elements in the vicinity of a typical $E_{C}$ fluctuation, $N \approx 124$. Coulomb matrix elements are calculated using the kernel of Poisson's equation from the self-consistent calculation. Thus screening by the gates is included automatically [12]. We have identified four stable orbits for this device of which two are observable in Fig. 2. Here $n_{62}=2\left(n_{p} \equiv\right.$ occupancy of state $\left.p\right)$ and $n_{63}=0$. The grey scale plot of Coulomb matrix elements shows that this state, as well as $p=60$, another scar along a slightly different orbit, have higher values of $U_{p}$ [13]. Scars occur in families as additional nodes are added along the same orbit (compare $p=62$ and $p=50$ and note their interaction). The Coulomb matrix elements given here
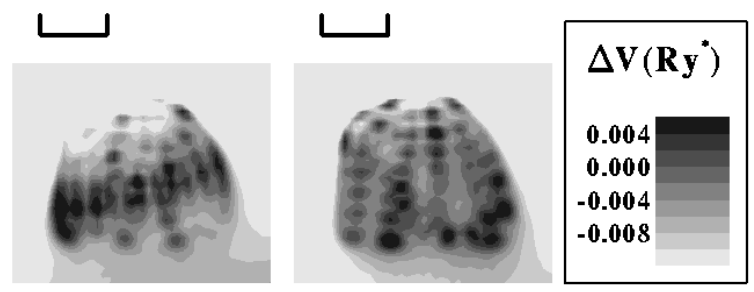

FIG. 3. Potential difference contours showing influence of $p=62$, scar state filling (left) and that of more chaotic state $p=64$ (cf. Fig. 2). Plunger gates indicated.

are between self-consistent states 14$]$. Thus, even though $W_{62,63}$ is relatively small $\left(\sim 0.18 R y^{*}\right)$, it is the interaction of these two states which produces the gap at $E_{F}$ and the fluctuation of $E_{C}$ at this $N$. The strong 1-d scar $p=62$ at $E_{F}$ acts like a single atomic valence electron and strongly influences the potential (Fig. 3), creating a central "ridge" which modifies $p=63$, reducing the Coulomb interaction between $p=62$ and $p=63$.

A comparison between our calculations and the experimental results of Sivan et al. [2] shows a striking discrepancy. While the gate voltage spacing fluctuations $\left(\Delta_{2}\right.$ in Ref. [2]) are symmetric, the fluctuations of $E_{C}$ computed here all proceed upward from a smoothly decreasing base. Furthermore, a comparison of the histograms of the fluctuations (not shown) reveals that the fluctuations in $E_{C}$ have an r.m.s. deviation of only about $5 \%$ of $E_{C}$, a result that is smaller by a factor of two or three from the data for $\Delta_{2}$. These discrepancies emphasize the importance, heretofore not fully appreciated, of distinguishing between the "inverse compressibility" (what we call the charging energy) at fixed $V_{g}$ and the gate voltage spacing (normalized by the capacitance ratio $\alpha$, see below) between Coulomb oscillations. These two quantities are equivalent only within the constant interaction model and, as we now show, small, upward variations in $E_{C}$ can produce much larger, symmetric variations in $\Delta V_{g}$.
To a reasonable approximation we can write $F\left(N, V_{g}\right)=\frac{1}{2} E_{C}\left(V_{g}\right) N^{2}+b\left(V_{g}\right) N+c$, i.e. $F$ is quadratic in $N$ at each $V_{g}$. In the CI model, $b\left(V_{g}\right)=-e \alpha V_{g}$ where $\alpha$ is the ratio of the dot-gate capacitance $C_{d g}$ to the dot selfcapacitance $C$, and $E_{C}=\frac{e^{2}}{C}+\sum_{p}^{N} \epsilon_{p}^{0}$ (at $\left.T=0\right)$. $C, \alpha$ and $\epsilon_{p}^{0}$ (the single particle level energies) are all assumed to be independent of $V_{g} . \Delta V_{g}$ is determined by writing $F\left(N, V_{g}\right)=F\left(N+1, V_{g}\right)$ and $F\left(N+1, V_{g}^{\prime}\right)=F\left(N+2, V_{g}^{\prime}\right)$ and subtracting, resulting in:

$$
e \alpha \Delta V_{g}=\frac{e^{2}}{C}+\epsilon_{N+2}^{0}-\epsilon_{N+1}^{0} .
$$

However when $C$, $\alpha$ and $\epsilon_{p}^{0}$ depend on $V_{g}$, the procedure for deriving Eq. 1 leads to

$$
\begin{aligned}
& e \alpha\left(V_{g}^{\prime}\right) V_{g}^{\prime}-e \alpha\left(V_{g}\right) V_{g}= \frac{(N+3 / 2) e^{2}}{C\left(V_{g}^{\prime}\right)} \\
&-\frac{(N+1 / 2) e^{2}}{C\left(V_{g}\right)}+\epsilon_{N+2}^{0}\left(V_{g}^{\prime}\right)-\epsilon_{N+1}^{0}\left(V_{g}\right) .
\end{aligned}
$$

When, in particular, $C$ fluctuates appreciably from $V_{g}$ to $V_{g}^{\prime}$ the influence on $\Delta V_{g}$ can be magnified, as illustrated in Fig. 4. Here we have calculated $F\left(N, V_{g}\right)$ on sufficiently fine grids to determine $\Delta V_{g}$ numerically.

To complete the picture we note that the frequency of scars in the spectrum, and hence the number of large oscillations in $E_{C}$, decrease with $N$. This is somewhat evident in Fig. 1 in that the spacing of oscillations increases with $N$. The scars are remnants of closed orbits and are thus effectively one dimensional. Their density of states (DOS) decreases as $1 / \sqrt{E}$, in contrast to the constant DOS for the 2D dot as a whole. Thus the overall frequency of scars in the spectrum will diminish. In addition, the extent to which a scar at $E_{F}$ pushes up the ensuing state should also diminish with $N$. (Again, the oscillations in Fig. 1 for larger $N$ appear to grow smaller). The reason is twofold. First, screening by the dot electrons increases with $N$. Second, the influence of the inhomogeneous potential fluctuation produced by the scar on the energy of a typical chaotic state should depend on the wavelength of that state $\lambda$ in comparison to the dot size, i.e. the kinetic energy cost of avoiding the scar decreases with $\lambda$.

When one considers larger dots with complicated scars occupying much of the dot area, the distinction between scars and non-scars, in terms of Coulomb matrix elements, tends to disappear [15]. In this case the fluctuations in $E_{C}$ will depend on the dot size, the fraction of the dot area occupied by the states near $E_{F}$, the screening length $\lambda_{s c}$ and perhaps dot geometry.

Sivan et al. found that while the fluctuations in $e \alpha \Delta V_{g}$ were much greater than the average single particle level spacing $\Delta$, the temperature dependence of these fluctuations was, seemingly, governed by $\Delta / k_{B} T$ [2]. We have argued that the fluctuations in $E_{C}$ should decrease with $N$ due to decreasing frequency of scars and their 


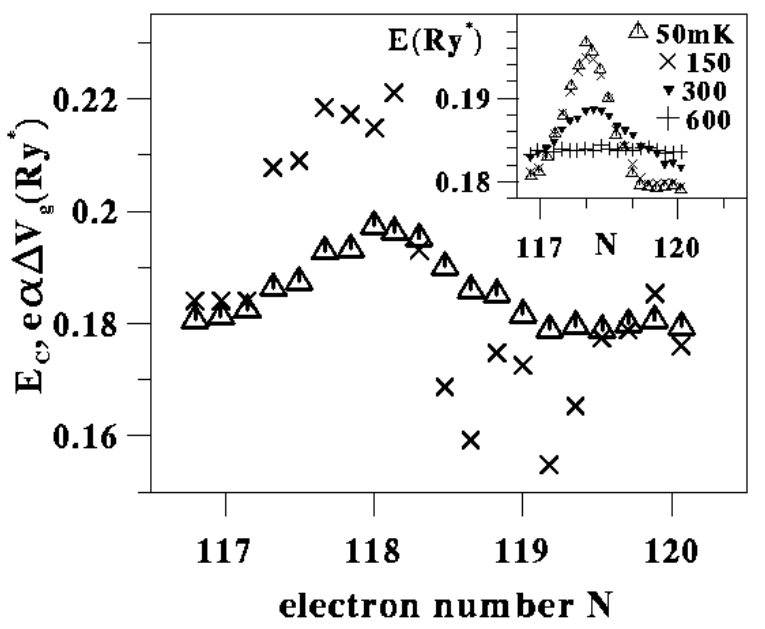

FIG. 4. Charging energy and gate voltage spacing vs $N$. Non-plunger gates differ from preceding plots, giving large fluctuation at $N=118$. While $E_{C}$ fluctuation is purely upward, fluctuation in $V_{g}$ spacing is symmetric and substantially greater in magnitude. Inset: $E_{C}$ fluctuation for various $T$. Fluctuations vanish far below $k_{B} T=E_{C}$.

decreasing influence on neighboring states. We cannot say definitively, however, whether these fluctuations should scale with $\Delta$ (dot area) or with $E_{C}$ (dot radius), though since $\lambda_{s c} \sim \Delta^{-1}$, the former seems more likely in the large dot limit. Empirically, however, the fluctuations in $E_{C}$ are comparable to $\Delta$ and, as noted, the enhancement of fluctuations in $\Delta V_{g}$ (Eq. 2) derives from $V_{g}$ dependence of capacitances and energy levels. Consequently, (Fig. 4 inset), the fluctuations essentially vanish by $600 \mathrm{mK}(\approx 0.75 \Delta)$ even though this is a factor of twenty smaller than $E_{C}$ itself.

Finally, in recent studies by Patel et al., citation in [7], no correlation was found to exist between peak heights and spacings. We propose that a stadium geometry, with quantum point contacts (QPCs) placed along the long axis, should indeed show this correlation. Certainly one stable periodic orbit will be that which simply oscillates along the central axis, so that these states will give greatly enhanced peak heights. Since, according to our discussion, scars associated with this orbit will float at $E_{F}$ for certain ranges of $V_{g}$, one should find corresponding groups of large Coulomb oscillations separated by a large $V_{g}$ spacing from neighboring oscillations.

In conclusion, we have demonstrated that strongly scarred, quasi-1d states, by virtue of their strong Coulomb interactions, produce quasi-periodic fluctuations in the charging energy of chaotic quantum dots. We have shown that the scale of those fluctuations is consistent with the single particle level spacing, but that fluctuations in gate voltage spacings of Coulomb oscillations are, due to $V_{g}$ dependence of capacitances and energy levels, substantially greater and differently (symmetrically) distributed. Finally, we have proposed a simple exper- imental arrangement which could elucidate the role of self-consistency in chaotic systems discussed herein.

I wish to thank Dr. Uri Sivan for providing me with details of his devices and measurement. I wish to acknowledge a particularly helpful conversation with Oded Agam. I have also had helpful conversations with Y. Alhassid and co-workers, S. Das Sarma, F. Stern, C. Marcus, S. Patel, N. Wingreen Y. Aoyagi and T. Sugano. Support from Riken Computing Center - Fujitsu VPP500 Supercomputer are gratefully acknowledged. This work supported by grants from the Japan Ministries of Education and Science and Technology.

[1] T. A. Brody et al. Rev. Mod. Phys. 53, 385 (1981); F. Haake, Quantum Signatures of Chaos (Springer, Berlin, 1991).

[2] U. Sivan et al. Phys. Rev. Lett. 77, 1123 (1996).

[3] M. Stopa, Phys. Rev. B, 54, 13767 (1996).

[4] J. A. Folk it et al. Phys. Rev. Lett. 76, 1699 (1996).

[5] R. Berkovits and Y. Avishai, J. Phys. Condens. Matter 8389 (1996).

[6] Ya.M. Blanter et al. Phys. Rev. Lett. 78, 2449 (1997).

[7] This explains the absence of an even-odd symmetry which would be expected in a spin degenerate version of the CI model, S. R. Patel et al., preprint, condmat/9708090.

[8] The self-consistent tendency for multiple levels to be partially occupied near $E_{F}$ for non-zero $T$ also provides a physical explanation for unexpected correlation of peak heights; see M. Stopa, Phys. Rev. B 48, 18340 (1993) and, more recently, J. A. Folk et al., Phys. Rev. Lett. 76, 1699 (1996).

[9] D. Jovanovic and J. P. Leburton, Phys. Rev B, 49, 7474 (1994).

[10] J. C. Slater, The Self-Consistent Field for Molecules and Solids, McGraw-Hill, New York, 1974. More recent techniques on elimination of self-interaction effects in DF theory are discussed in M. M. Rieger and P. Vogl, Phys. Rev. B 5216567 (1995) and references therein.

[11] P. I. Tamborenea, R. J. Radtke and S. Das Sarma, preprint, cond-mat/9604099.

[12] M. Stopa, (to be published).

[13] We note that the application of a magnetic field $B$, by reducing the effective dimensionality of all eigenstates also imparts structure to the Coulomb matrix elements. The influence of $B$ on the spectrum can be seen in Ref. 3, Fig. 13.

[14] This is to be distinguished from the Coulomb matrix elements, between bare levels, screened by the dot electrons themselves, as determined, for example, via RPA.

[15] For larger $N$ one expects scars to be associated with groups of eigenstates rather than our small dot case where they are represented by single eigenfunctions. 